\title{
Hard X-ray search for unidentified EGRET sources in the BAT survey archive
}

\author{
V. Mangano ${ }^{* a}$ A. Segreto ${ }^{a}$, G. Cusumano ${ }^{a}$, V. La Parola ${ }^{a}$, C. Ferrigno ${ }^{b}$, P. Romano ${ }^{a}$, \\ S. Campana ${ }^{c}$, G. Chincarini ${ }^{c}$, G. Tagliaferri ${ }^{c}$, P. Giommi $^{e}$ \\ ${ }^{a}$ INAF, Istituto di Astofisica Spaziale e Fisica Cosmica \\ Via U. La Malfa 153, I-90146 Palermo, Italy \\ ${ }^{b}$ Institut für Astronomie und Astrophysik Tübingen (IAAT) and \\ ISDC, Data Centre for Astrophysics \\ Chemin d'Ècogia 16, CH-1290 Versoix, Switzerland \\ ${ }^{c}$ INAF, Osservatorio Astronomico di Brera \\ via E. Bianchi 46, I-23807 Merate, Italy \\ ${ }^{d}$ Università degli Studi di Milano, Bicocca \\ Piazza delle Scienze 3, I-20126 Milano, Italy \\ e ASDC, ASI Science Data Center \\ via Galileo Galilei, I-00044 Frascati, Italy \\ E-mail: vanessa@ifc.inaf.it
}

\begin{abstract}
The third EGRET catalogue includes a large number of unidentified sources. Some of these objects may represent a new class of yet undiscovered gamma-ray sources. Using a software tool developed for the Swift-BAT survey data analysis, the Bat Imager, highly efficient and independent from the tasks distributed by the Swift-BAT hardware team, we searched the archive of the first 39 months of Swift observations in the hard X-ray energy band, 14-150 keV, to find the counterparts of the unidentified EGRET sources, and then study their broad band emission properties. We found 23 associations, 20 of them already known in the literature and 3 newly proposed. A check of our set of associations with the revised EGRET catalogue [4] confirms only 15 of them, but gives 3 new associations, one with an EGRET source detected for the first time in the revised catalogue. We plan to investigate the proposed associations through exploitation of multi-wavelength archival data and follow-up observations.
\end{abstract}

7th INTEGRAL Workshop

September 8-11 2008

Copenhagen, Denmark

${ }^{*}$ Speaker. 


\section{Introduction}

Surveys are the most effective way to make important steps beyond the study of objects with known properties based on observations in other bands, because they allow to discover new classes of objects and astrophysical phenomena. They are also essential for population and evolution studies, that require the knowledge of complete samples of sources.

In the high energy gamma-rays, the most extended survey up to date has been performed between 1991 and 1995 by EGRET (Energetic Gamma Ray Experiment Telescope), the gammaray telescope on the Compton Gamma Ray Observatory (CGRO), sensitive in the energy range from $30 \mathrm{MeV}$ to over $20 \mathrm{GeV}$. The third EGRET catalogue [11] includes 271 sources detected at energies $>100 \mathrm{MeV}$, and $\sim 170$ of them are still unidentified, lacking counterparts at lower wavelengths.

Surveys in the hard X-ray band (10-600 keV) have been poorly developed for decades since the most extended to date was conducted by the HEAO-A4 experiment in the late seventies [13]. Recently, two more all-sky surveys at hard X-ray energies (about 20-200 keV) have effectively been carried out: the INTEGRAL-IBIS survey [18, 3] and the Swift-BAT survey [10, 2]. The former, started in 2002, was initially devoted mostly to the Galactic plane scan, but has now reached a $>70 \%$ coverage of the sky with an exposure of at least $10 \mathrm{ks}$. It provided the detection of more than 500 (mostly Galactic) sources in the $20-100 \mathrm{keV}$ band. The latter, started in late 2004, had from the very beginning an almost uniform sky coverage because of the very large BAT field of view (FOV; $\sim 2$ sr, partially coded, compared with the 0.02 sr IBIS FOV), and the almost-random pointing observation strategy related to the Gamma-ray burst chase which is the main purpose of the Swift mission. These characteristics make the Swift-BAT survey the natural complement to the INTEGRAL survey at high Galactic latitude.

The preliminary results of the first 3 months of the Swift-BAT survey data have been published by [14]. An extended analysis of 9 months of data has been performed by [17] and on 1/8th of the sky, by [1]]. The largest published catalogue of BAT detected sources (presented in [17]) includes only 150 AGNs at high Galactic latitude (at more than 5 degrees from the Galactic Plane). We developed a dedicated software suite [15, 16], completely independent from the one developed by the Swift-BAT hardware team, to analyze BAT survey data, optimized for maximum speed and minimum CPU load in all-sky source detection, and run it over the first 39 months of Swift-BAT observations. We detected a total of 968 sources above the $4.8 \sigma$ significance limit. Through crosscorrelation with the Simbad databases and published hard X-ray catalogues, and through analysis of field observations of soft X-ray instruments we obtained a catalogue of 748 identified sources, presented in [1, 12] and fully discussed in [6].

The main goal of this work is to identify potential hard X-rays counterparts of EGRET sources in the new BAT survey catalogue we produced, which is the most extended to date.

\section{Results}

We performed a cross correlation of the Third EGRET catalogue with our 39 months BAT survey catalogue, consisting of 748 identified sources. We accepted as possible counterpart of an EGRET source the nearest BAT source falling within the EGRET error radius, that we assumed 
given by $\theta_{95}$ in [1], i.e. the radius of the circle containing the same solid angle as the $95 \%$ confidence contour. We obtained 23 associations. The 23 images in Fig. 1 1 and Fig. 目 show the $3 \times 2$ $\mathrm{deg}^{2}$ sky regions centred on these EGRET sources (red circles, with corresponding EGRET error radius) and their counterparts (green circles, with radius set to 5 arcmin for better visualization).

We evaluated the fraction of expected spurious identifications due to spatial coincidence producing a set of 271 coordinate pairs by shifting the positions of the EGRET sources of 5 degrees in diagonal direction with respect to the Galactic reference system and cross correlating them with the BAT survey catalogue. We obtained 7 spurious associations.

In Table 1 we show all relevant information about the BAT fluxes and hardness ratios of the counterparts, when available. According to the Third EGRET catalogue, sources 12, 15, 16, 17, and 18 are unidentified. The associations here include two AGNs, two Galactic accreting binary systems, a cluster of galaxies, and an unidentified INTEGRAL source.

The association of source 15 (3EG J1639-4702) with IGR J16393-4643 has already been proposed in [5]. However this EGRET source has also been recently proposed for association with the Supernova remnant G338.3-0.0 detected by HESS [9].

The association of source 17 (3EG J1736-2908) with the active galactic nucleus GRS 1734-292 has already been discussed in [8].

The reliability of the association of source 18 (3EG J1746-2851) to the low mass X-ray binary 1E $1743.1-2852$ is to be better investigated because the very same source has also been proposed for association with the cluster of massive stars, G0.12+0.017 [19].

Associations of source 12 (3EG J1424+3734) to the cluster of galaxies A1914 and of source 16 (3EG J1734-3232) to the unidentified INTEGRAL source IGR J17354-3255 are new.

Recently, Casandjian \& Grenier (2008) produced a revised catalogue of point $\gamma$-ray sources detected by EGRET above $100 \mathrm{MeV}$, reprocessing the entire data set and using new Galactic interstellar emission models [4]. Their catalogue contains 188 sources, mostly corresponding to the identified sources in the Third EGRET catalogue. According to the revised positions and $\theta_{95}$ values of EGRET sources in the Casandjian \& Grenier (2008) catalogue, the associations of sources 5, 10, $11,16,17,20$ and 22 would not be confirmed. Moreover, the EGRET source 3EG J1639-4702 (source 15) has no counterpart in the Casandjian \& Grenier (2008) catalogue. A cross correlation of the Casandjian \& Grenier (2008) catalogue with our 39 months BAT survey catalogue gives a total of 18 associations, 15 of them already listed in Table 1. plus the following:

3EG J0241+6103/EGR J0240+6112/GT 0236+610, 3EG J0533-6916/EGR J0537-6946/LMC X-1, and EGR J1642+3940/4C39.48, where the first two are associations well known in the literature and the last one refers to a new source with no counterpart in the Third EGRET catalogue. The number of expected spurious associations, evaluated with the same procedure used with the Third EGRET catalogue is of 5 . 

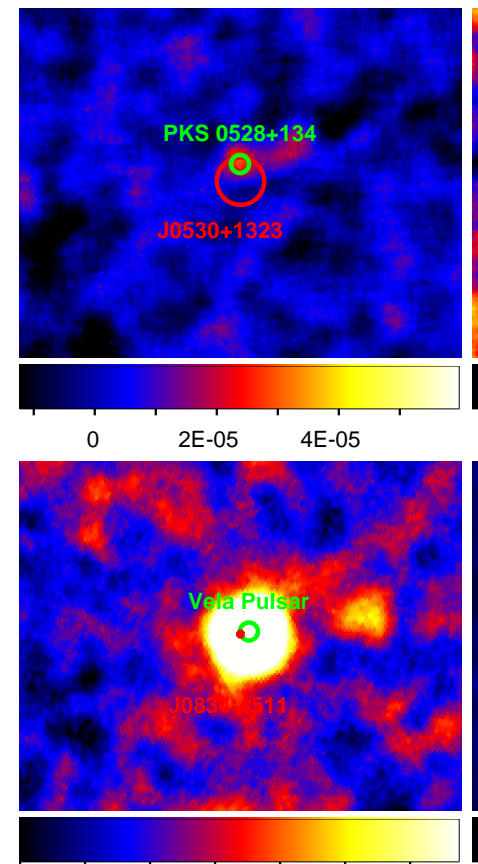

05
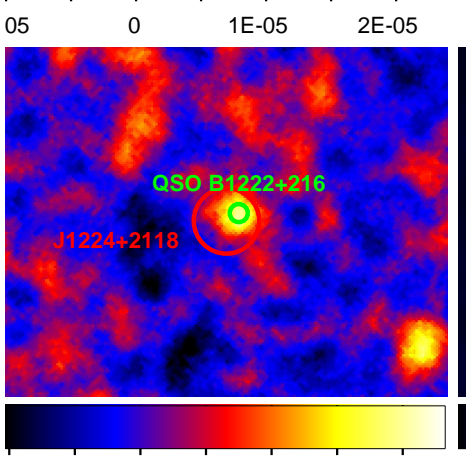

05

0

1E-05

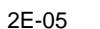

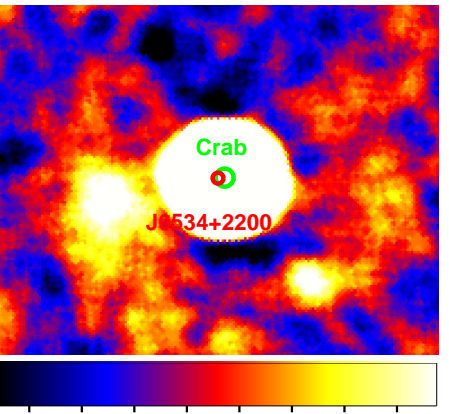

$-1 \mathrm{E}-05$
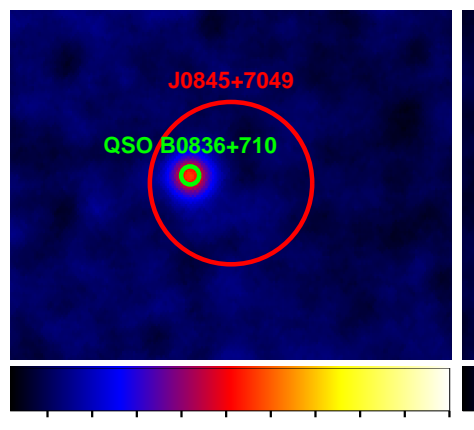

0.00012

$0.000 \quad 0$
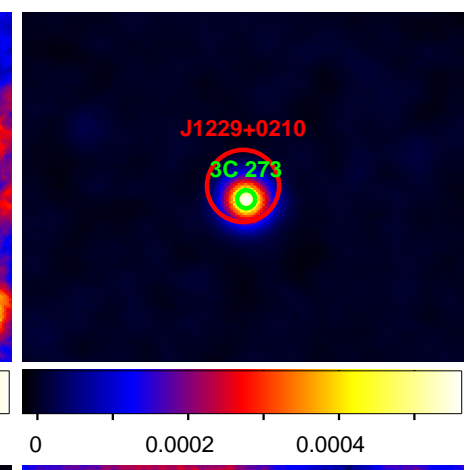

0.0002

0.0004
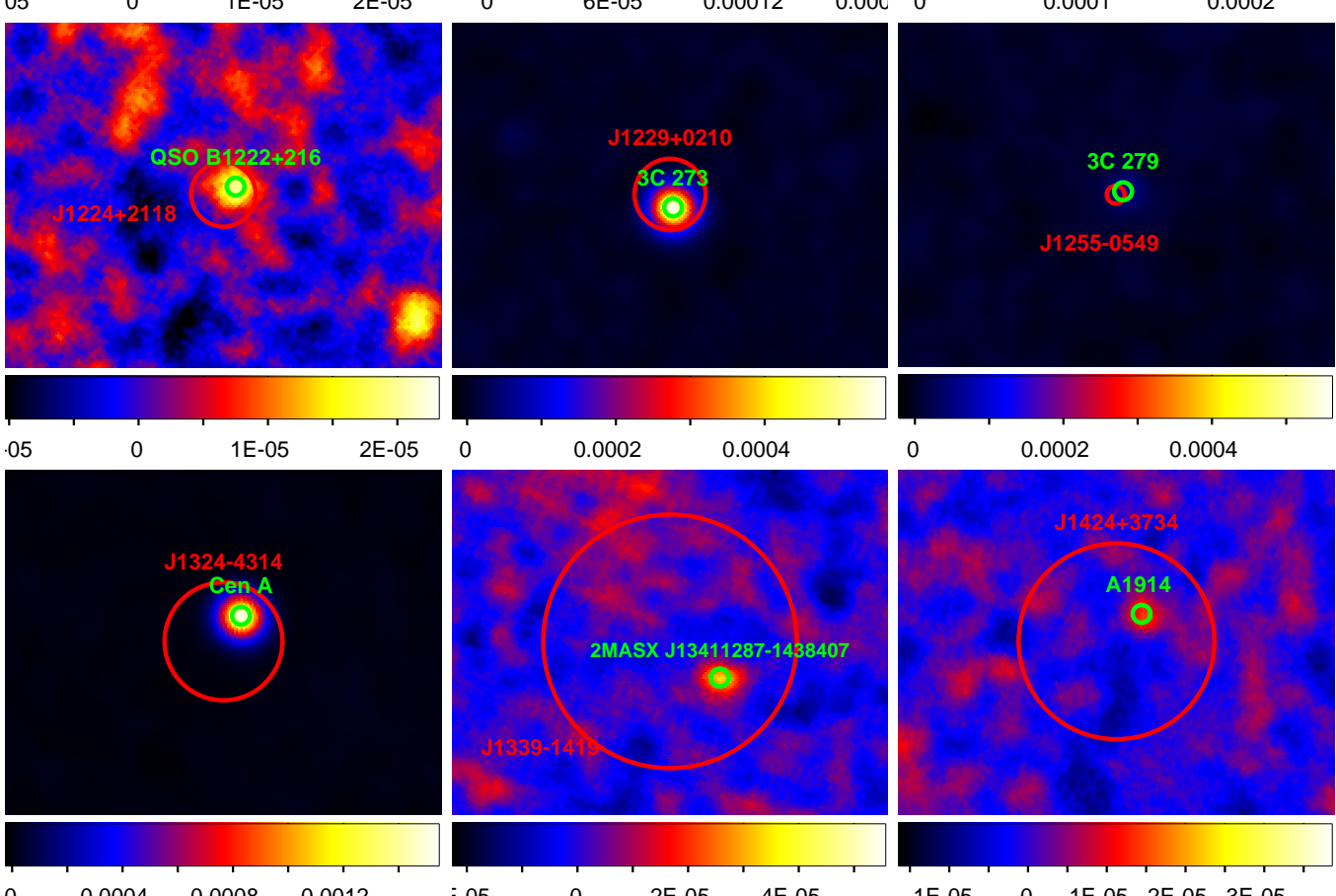

0

0.0002

0.0004

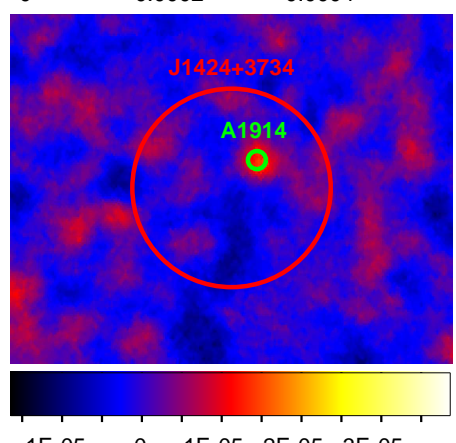

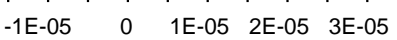

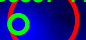

$0 \quad 2 \mathrm{E}-05 \quad 4 \mathrm{E}-05 \quad 6 \mathrm{E}-05 \quad 8 \mathrm{E}-05$

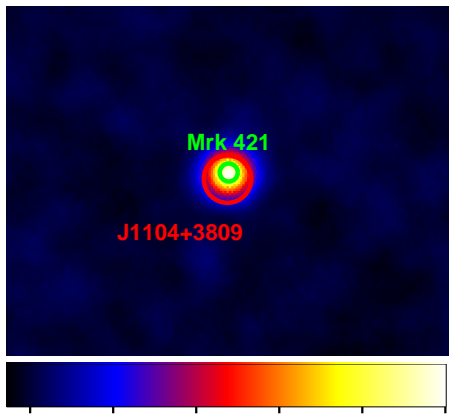

0002 

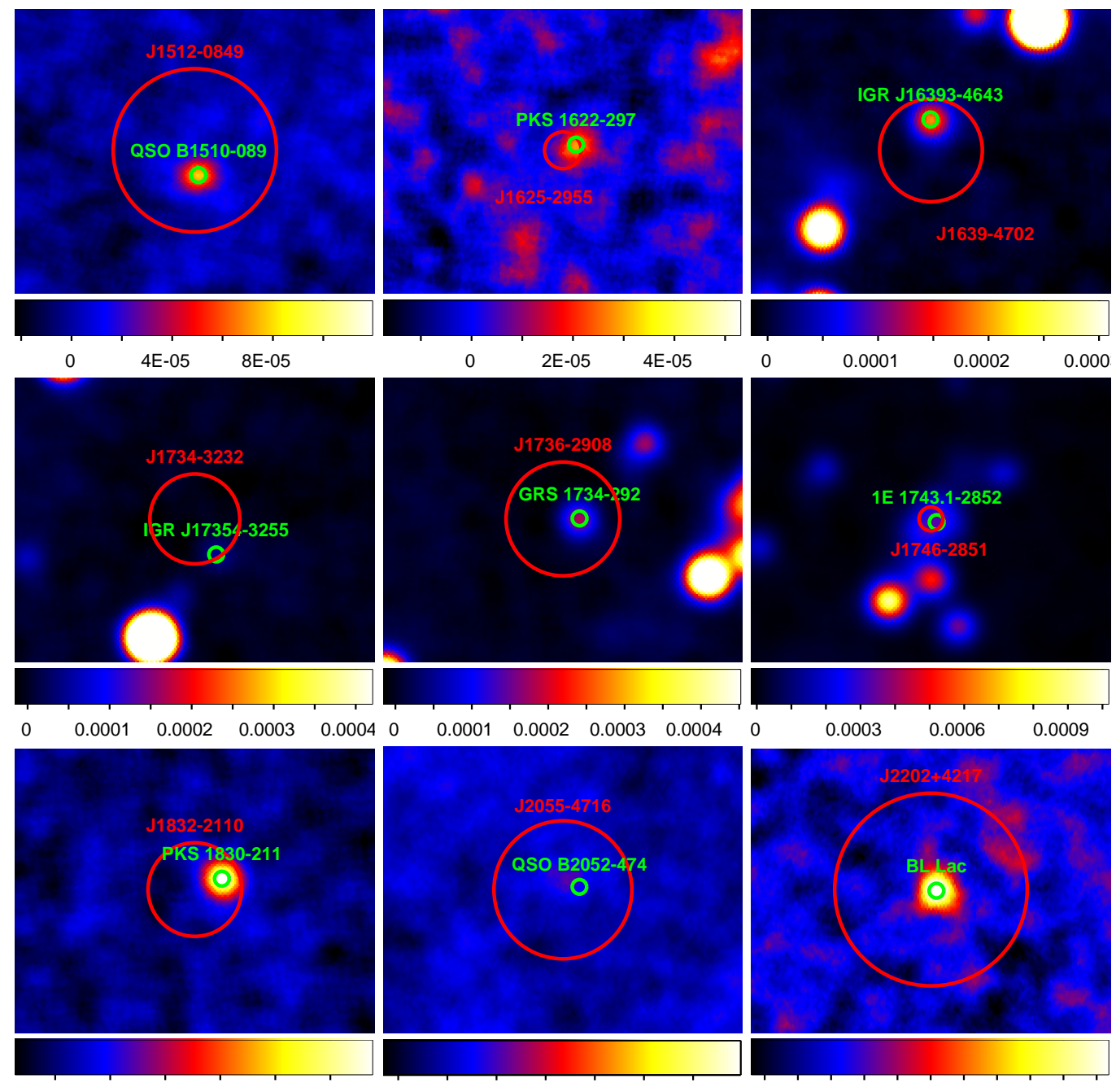

$\begin{array}{llll}0.0001 & 0.0002 & 0.0003 & 0.0004\end{array}$

$0 \quad 4 \mathrm{E}-05$

$8 \mathrm{E}-05$
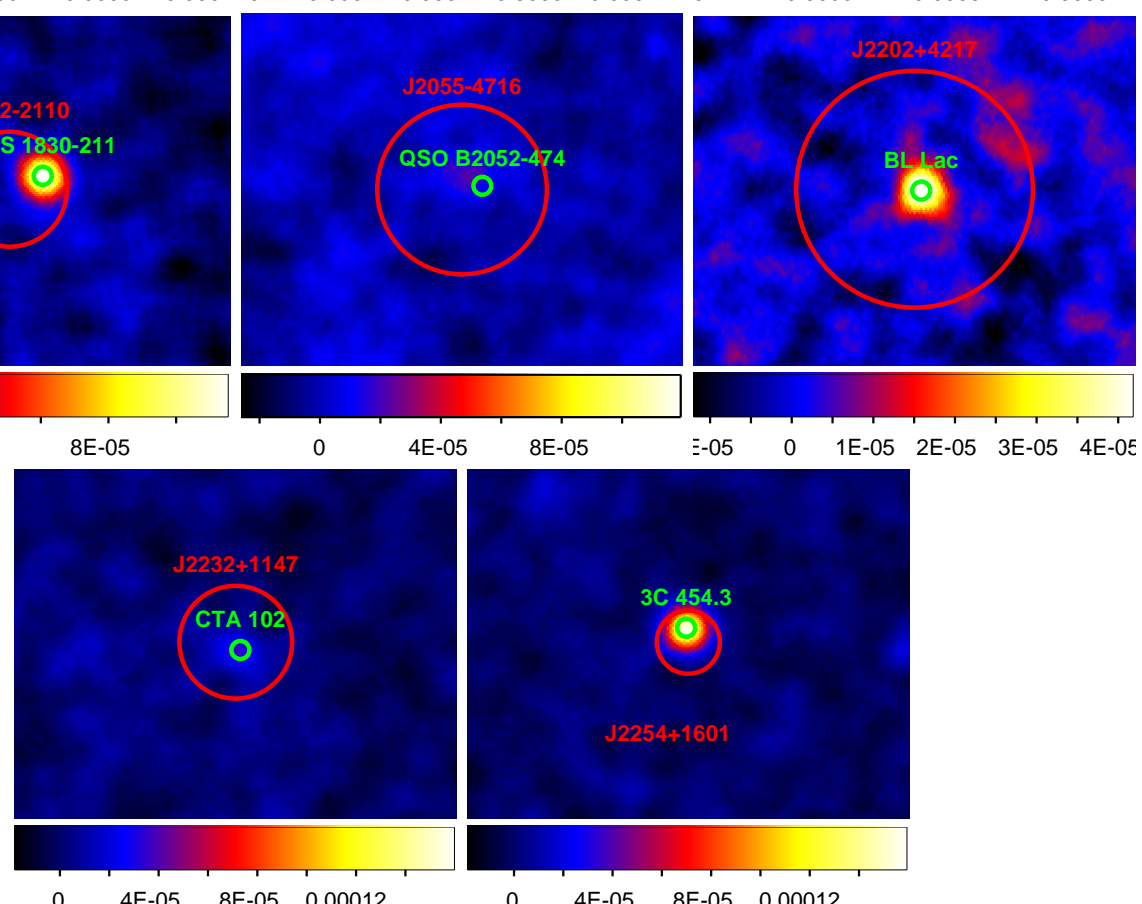

Figure 2: Same as Fig. 11 for EGRET sources with counterparts 13-23 in Table 1 . 
Table 1: Counterparts for EGRET sources in the BAT survey.

\begin{tabular}{|c|c|c|c|c|c|c|c|c|c|}
\hline ID & Name & $\begin{array}{l}\text { RA } \\
(\mathrm{deg}) \\
\end{array}$ & $\begin{array}{l}\text { Dec } \\
(\mathrm{deg}) \\
\end{array}$ & $\begin{array}{l}\theta_{95^{\mathrm{a}}} \\
(\mathrm{deg}) \\
\end{array}$ & Alt. Name & Type $^{b}$ & $\sigma_{14-150 \mathrm{keV}}$ & $\begin{array}{l}\text { Flux }_{14-150 \mathrm{keV}} \\
\left(10^{-11} \mathrm{erg} \mathrm{cm}^{-2} \mathrm{~s}^{-1}\right)\end{array}$ & $\begin{array}{l}\text { Hardness } \\
\left(\text { rate }_{30-150 \mathrm{keV}} / \text { rate }_{14-30 \mathrm{keV}}\right.\end{array}$ \\
\hline 1 & 3EG J0530+1323 & 082.74 & +13.38 & 0.210 & PKS 0528+134 & QSO & 4.66 & $1.53 \pm 0.33$ & - \\
\hline 2 & 3 EG J0534+2200 & 083.57 & +22.01 & 0.048 & Crab & Psr & 4670.65 & $2111.51 \neq 0.45$ & $0.80016 \pm 0.0002$ \\
\hline 3 & 3EG J0540-4402 & 085.02 & -44.05 & 0.300 & QSO B0537-441 & BLL & 4.72 & $0.78 \pm 0.19$ & - \\
\hline 4 & 3EG J0834-4511 & 128.73 & -45.20 & 0.021 & Vela Pulsar & Psr & 48.50 & $12.20 \pm 0.25$ & $0.94 \pm 0.03$ \\
\hline 5 & 3EG J0845+7049 & 131.46 & +70.83 & 0.720 & QSO B0836+710 & QSO & 23.06 & $4.42 \pm 0.19$ & $1.40 \pm 0.12$ \\
\hline 6 & 3EG J1104+3809 & 166.10 & +38.15 & 0.210 & Mrk 421 & BLL & 62.78 & $11.41 \pm 0.18$ & $0.42 \pm 0.01$ \\
\hline 7 & $3 E G ~ J 1224+2118$ & 186.11 & +21.31 & 0.290 & QSO B1222+216 & QSO & 5.27 & $0.97 \pm 0.18$ & - \\
\hline 8 & $3 E G ~ J 1229+0210$ & 187.25 & +02.17 & 0.320 & $3 C 273$ & QSO & 105.20 & $26.86 \pm 0.26$ & $1.18 \pm 0.02$ \\
\hline 9 & 3EG J1255-0549 & 193.98 & -05.82 & 0.080 & 3C 279 & QSO & 10.41 & $2.66 \pm 0.26$ & $0.82 \pm 0.13$ \\
\hline 10 & 3EG J1324-4314 & 201.15 & -43.25 & 0.530 & Cen A & Sy2 & 219.76 & $75.59 \pm 0.34$ & $1.03 \pm 0.01$ \\
\hline 11 & 3EG J1339-1419 & 204.84 & -14.32 & 1.140 & 2MASX J13411287-1438407 & Sy1 & 6.58 & $1.75 \pm 0,27$ & - \\
\hline $12^{\mathrm{c}}$ & 3EG J1424+3734 & 216.22 & +37.58 & 0.880 & A1914 & $\mathrm{ClG}$ & 5.09 & $0.40 \pm 0,08$ & - \\
\hline 13 & 3EG J1512-0849 & 228.17 & -08.83 & 0.890 & QSO B1510-089 & QSO & 10.75 & $3.26 \pm 0,30$ & $1.51 \pm 0.30$ \\
\hline 14 & 3EG J1625-2955 & 246.36 & -29.92 & 0.200 & PKS 1622-297 & QSO & 4.65 & $1.73 \pm 0.39$ & - \\
\hline 15 & 3EG J1639-4702 & 249.78 & -47.04 & 0.560 & IGR J16393-4643 & HXB & 19.71 & $9.48 \pm 0.48$ & $0.12 \pm 0.01$ \\
\hline 16 & 3EG J1734-3232 & 263.56 & -32.55 & 0.490 & IGR J17354-3255 & gam & 5.69 & $2.98 \pm 0.54$ & $0.39 \pm 0.10$ \\
\hline 17 & 3EG J1736-2908 & 264.16 & -29.14 & 0.620 & GRS 1734-292 & Sy1 & 16.82 & $9.08 \pm 0.54$ & $0.81 \pm 0.08$ \\
\hline 18 & 3EG J1746-2851 & 266.51 & -28.86 & 0.130 & 1E 1743.1-2852 & LXB & 28.51 & $15.38 \pm 0.54$ & $0.43 \pm 0.02$ \\
\hline 19 & 3EG J1832-2110 & 278.10 & -21.18 & 0.510 & PKS 1830-211 & QSO & 11.40 & $5.35 \pm 0.48$ & $1.48 \pm 0.27$ \\
\hline 20 & $3 E G$ J2055-4716 & 313.80 & -47.28 & 0.760 & QSO B2052-474 & QSO & 5.00 & $1.43 \pm 0.29$ & - \\
\hline 21 & $3 E G ~ J 2202+4217$ & 330.60 & +42.29 & 1.050 & B1 Lac & BLL & 7.39 & $1.86 \pm 0.25$ & $0.90 \pm 0.19$ \\
\hline 22 & $3 E G ~ J 2232+1147$ & 338.11 & +11.80 & 0.500 & CTA 102 & QSO & 7.91 & $1.86 \pm 0.23$ & $1.14 \pm 0.26$ \\
\hline 23 & 3 EG J2254+1601 & 343.51 & +16.02 & 0.280 & $3 \mathrm{C} 454.3$ & QSO & 32.32 & $7.70 \pm 0.24$ & $1.44 \pm 0.08$ \\
\hline
\end{tabular}




\section{Summary}

We cross correlated the Third EGRET catalogue with the 39 months BAT survey catalogue [6, 7, 12] to look for possible hard X-ray counterparts of unidentified EGRET sources. We obtained 23 possible associations, 20 of them already known and 3 newly proposed. A cross correlation of the BAT catalogue with the revised EGRET catalogue [何] confirms only 15 of our associations, but gives 3 new associations, one of them with an EGRET source detected for the first time in the revised catalogue. The reliability of all the proposed associations must be carefully investigated. To this aim we plan to make use of data in other bands of the electromagnetic spectrum, either already existing in the literature or in the archives, or to be obtained in future observations.

\section{Acknowledgments}

This work was supported in Italy by the MIUR Grant CRA 1.06.10.07. Italian researchers acknowledge the support of Nature $(455,835-836)$ and thank the Editors for increasing the international awareness of the current critical situation of the Italian Research.

\section{References}

[1] Ajello, M., Greiner, J., Kanbach, G., et al., 2008, ApJ, 678, 102

[2] Barthelmy, S. D., Barbier, L. M., Cummings, J. R., et al., 2005, Space Science Review, 120, 143

[3] Bird, A. J., Malizia, A., Bazzano, A., et al., 2007, ApJ Supplement Series, 170, 175

[4] Casandjian, J.-M., \& Grenier, I. A. 2008, $A \& A, \mathbf{4 8 9}, 849$

[5] Combi, J. A., Ribó, M., Mirabel, I. F., \& Sugizaki, M. 2004, A\&A, 422, 1031

[6] Cusumano, G., Segreto, A., La Parola, V., et al., 2009, A\&A, submitted

[7] Cusumano, G., La Parola, V., Ferrigno, C., et al., 2009, in proceeding of 7th INTEGRAL Workshop, PoS (Integral08) 134

[8] Di Cocco, G., et al. 2004, A\&A, 425, 89

[9] Funk, S., Hinton, J. A., Pühlhofer, G., Aharonian, F. A., Hofmann, W., Reimer, O., \& Wagner, S. 2007, ApJ, 662, 517

[10] Gehrels, N., Chincarini, G., Giommi, P., et al., 2004, ApJ, 611, 1005

[11] Hartman, R.C., Bertsch, D.L., Bloom, S.D., et al., 199, ApJ, 123, 79

[12] La Parola, V., Cusumano, G., Segreto, A., et al., 2009, in proceeding of 7th INTEGRAL Workshop, PoS (Integral08) 96

[13] Levine, A. M., Lang, F. L., Lewin, W. H. G., et al., 1984, ApJ Supplement Series, 54, 581

[14] Markwardt, C. B., Tueller, J., Skinner, G. K., et al., 2005, ApJ, 633, L77

[15] Segreto, A., et al., 2009, in proceeding of 7th INTEGRAL Workshop, PoS (Integra 108) 131

[16] Segreto, A., Cusumano, G., Ferrigno, C., et al., 2009, $A \& A$, submitted

[17] Tueller, J., Mushotzky, R. F., Barthelmy, S., et al., 2008, ApJ, 681, 113

[18] Ubertini, P., Lebrun, F., Di Cocco, G., et al., 2003, A\&A, 411, L131

[19] Yusef-Zadeh, F., Nord, M., Wardle, M., Law, C., Lang, C., \& Lazio, T. J. W. 2003, ApJ, 590, L103 